Trauma Berufskrankh 2010 · 12[Suppl 2]:96-103 DOI 10.1007/s10039-010-1614-y

Online publiziert: 8. April 2010

(c) Springer-Verlag 2010

\section{F. Bonnaire $\cdot$ P. Bula}

Klinik für Unfall-, Wiederherstellungs- und Handchirurgie, Städtisches Klinikum

Dresden-Friedrichstadt, Akademisches Lehrkrankenhaus, TU Dresden

\title{
Distale Humerusfrakturen
}

\section{Diagnostik, Klassifikation und Behandlungsstrategie}

\section{Epidemiologie}

Distale Humerusfrakturen waren lange eine Domäne der Kinder und Jugendlichen sowie oft Folge von Übermut und Leichtsinn bei jungen Männern (so genanntes Manta-Syndrom). In einer britischen Studie war die Häufigkeit dieser Frakturentität bei den über 12-Jährigen mit 5,7:100.00o Einwohnern im Jahr ermittelt worden [21]. Zunehmend häufiger treten diese Verletzungen bei Erwachsenen und insbesondere älteren Frauen auf. Der Anteil der distalen Humerusfrakturen wird auf insgesamt 2\% aller Frakturen des Erwachsenenalters geschätzt [24].

Bei Kindern ist die suprakondyläre $\mathrm{Hu}$ merusfraktur mit 3\% aller Brüche die häufigste Fraktur über dem Ellenbogen. Die zweithäufigste Fraktur ist der Bruch des Condylus radialis, der das Ellenbogengelenk mit einbezieht. Die Brüche im Kindesalter sind oft Resultat eines Sturzes aus größerer Höhe oder eines direkten Auf- schlags auf den gebeugten Ellenbogen. Bei den jüngeren Erwachsenen gehen die distalen Humerusfrakturen eher auf Hochenergietraumen zurück [21].

\section{Klassifikationen}

Historisch werden die Frakturen am distalen Humerus eingeteilt in:

- suprakondyläre Frakturen

- transkondyläre Frakturen

- interkondyläre Frakturen

- Frakturen der Kondylen (medial und lateral)

- Frakturen der Gelenkfläche (Capitulum humeri und Trochlea)

- Epikondylenfrakturen

Die Brüche der Kinder sind in der Regel Extensions- oder Flexionsfrakturen, die durch einen Sturz auf den ausgestreckten oder gebeugten Arm hervorgerufen werden. Es sind nahezu immer suprakondyläre Frakturen, die aufgrund ih-

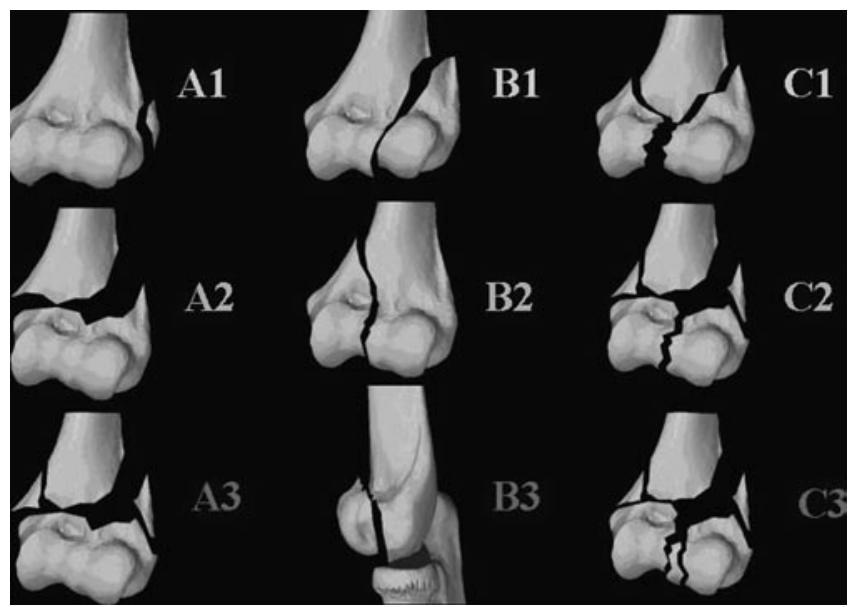

Abb. $1<$ AO-Klassifikation der distalen $\mathrm{Hu}$ merusfrakturen res Verschiebungsgrades eingeteilt werden (• Tab. 1).

Die distalen Humerusfrakturen des Erwachsenen werden nach AO [25] klassifiziert (• Abb. 1, $\bullet$ Tab. 2). Die mehrfragmentären Gelenkfrakturen vom Typ ${ }_{13} \mathrm{C}_{3}$ stellen immer eine Herausforderung für das versorgende Operationsteam dar.

Die operative Behandlung hat in Deutschland gegenüber den konservativen Verfahren v. a. bei den Frakturen im Erwachsenenalter zunehmend den Vorzug erhalten. Lediglich wenig dislozierte Frakturen werden konservativ mittels Oberarmgips behandelt. Nahezu alle Gelenkfrakturen, insbesondere bei Dislokation, werden mittels offener Reposition und übungsstabiler Osteosynthese behandelt, sodass postoperativ frühzeitig Bewegungsübungen möglich sind.

\section{Diagnostik}

Die klinische Diagnostik beginnt immer mit der Anamnese und der Abklärung des Unfallmechanismus. Die Beurteilung der Weichteilsituation bezüglich Hämatombildung, Deformierung, drohender Perforationen, offenen Weichteilläsionen und Begleitverletzungen an der oberen Extremität schließt sich an. Durchblutung und Sensibilität sind exakt zu dokumentieren.

Zur primären Schmerztherapie eignet sich die Gabe von Paracetamol (Perfalgan $^{\oplus}$ ) i.v. in Form einer Kurzinfusion. Dann wird in der Regel eine Röntgenübersichtsaufnahme des Ellenbogens in 2 Ebenen im Beisein des Unfallchirurgen, der die Lagerung unterstützt und hält, durchgeführt. 
Tab. 1 Verschiebegradabhängige Einteilung der suprakondylären Frakturen

\begin{tabular}{|ll} 
Typ & Charakteristika \\
I & $\begin{array}{l}\text { Geringe, wenn überhaupt erkennbare } \\
\text { Dislokation }\end{array}$ \\
II & $\begin{array}{l}\text { Dorsaldislokation mit radiologisch } \\
\text { intakter dorsaler Kortikalis }\end{array}$ \\
III $\quad \begin{array}{l}\text { Komplette Dislokation des distalen } \\
\text { Fragments ohne Kontakt zwischen } \\
\text { den Fragmenten }\end{array}$ \\
\hline
\end{tabular}

Tab. 2 AO-Klassifikation der distalen

Humerusfrakturen

Typ Charakteristika

13A Extra- Apophysenavulsionen artikuläre Einfache metaphysäre

Frakturen Frakturen

Metaphysäre Trümmerfrakturen

13B Artikuläre Sagittale laterale Frakturen Kondylenfrakturen Sagittale mediale Kondylenfrakturen Frontale Frakturen des Gelenks

13C Frakturen Einfache Konfiguration beider auf Gelenkniveau

Gelenk- Metaphysär auf

pfeiler Gelenkniveau

Einfach mehrfragmentär auf Gelenkniveau Metaphysär mehrfragmentär auf Gelenkniveau

Kann eine Fraktur projektionsradiographisch nachgewiesen werden, sollte beim Erwachsenen eine Computertomographieuntersuchung (CT-Untersuchung) zur Behandlungsplanung erfolgen. Bei Kindern und in Ausnahmefällen auch bei Erwachsenen kann zur Reduktion der Strahlendosis oder bei Verdacht auf Bandverletzungen oder okkulte Frakturen eine Magnetresonanztomographie (MRT) indiziert sein.

\section{Therapie}

\section{Behandlungsalgorithmus}

In der Regel ist mit der Diagnose einer Fraktur beim Erwachsenen auch die Indikation zur operativen Stabilisierung gegeben. Dies gilt sowohl für die intra- als auch die extraartikulären Verletzungen. Gründe hierfür sind die schlechte Heilungstendenz am distalen Humerus bei konservativer Behandlung und die in diesem Fall notwendige lange Ruhigstellung, die im-

Trauma Berufskrankh 2010 - 12[Suppl 2]:96-103 DOI 10.1007/s10039-010-1614-y

(c) Springer-Verlag 2010

\section{F. Bonnaire $\cdot$ P. Bula}

\section{Distale Humerusfrakturen. Diagnostik, Klassifikation und Behandlungsstrategie}

\section{Zusammenfassung}

Bedingt durch die komplexe Anatomie des Ellenbogengelenks stellen die distalen Frakturen des Humerus hohe Ansprüche an den behandelnden Unfallchirurgen. Voraussetzung für eine adäquate Therapie sind exakte Kenntnisse über den Gelenkaufbau und die Biomechanik. Ziel der Behandlung ist die Wiederherstellung der schmerzfreien, vollen Gelenkfunktion. Bedingung dafür sind die anatomische Reposition des Gelenkkomplexes, die Wiederherstellung der ligamentären Stabilität und eine möglichst übungsstabile Osteosynthese. Ein Problem stel-

\section{Distal fractures of the humerus. Diagnostic, classification and treatment strategy}

\section{Abstract}

Because of the complex anatomy of the elbow joint, distal fractures of the humerus present a great challenge to the attending trauma surgeon. A requirement for adequate treatment is the intimate knowledge of the joint-related structure and biomechanics. The aim of treatment is the restoration of a painfree entire range of motion. An essential condition for that is the anatomical reposition of the joint complex, restoration of the ligamentous stability and an exercise-stable osteo- len die zunehmend zu beobachtenden osteoporoseassoziierten Frakturen des älteren Menschen dar, welche häufig ein komplexes Frakturmuster beinhalten und aufgrund der schlechten Knochenqualität nicht selten primär oder sekundär eine endoprothetische Versorgung erforderlich machen.

\section{Schlüsselwörter}

Distale Humerusfraktur · Klassifikation . Behandlungsstrategie - Osteosynthese Komplikationen synthesis. A further problem is the increasing number of osteoporosis-related fractures of the distal humerus in the elderly. These fractures often cause complex osseous lesions that require endoprosthetic treatment primary or secondary.

\section{Keywords}

Distal humeral fractures - Classification . Treatment strategy - Osteosynthesis . Complications 

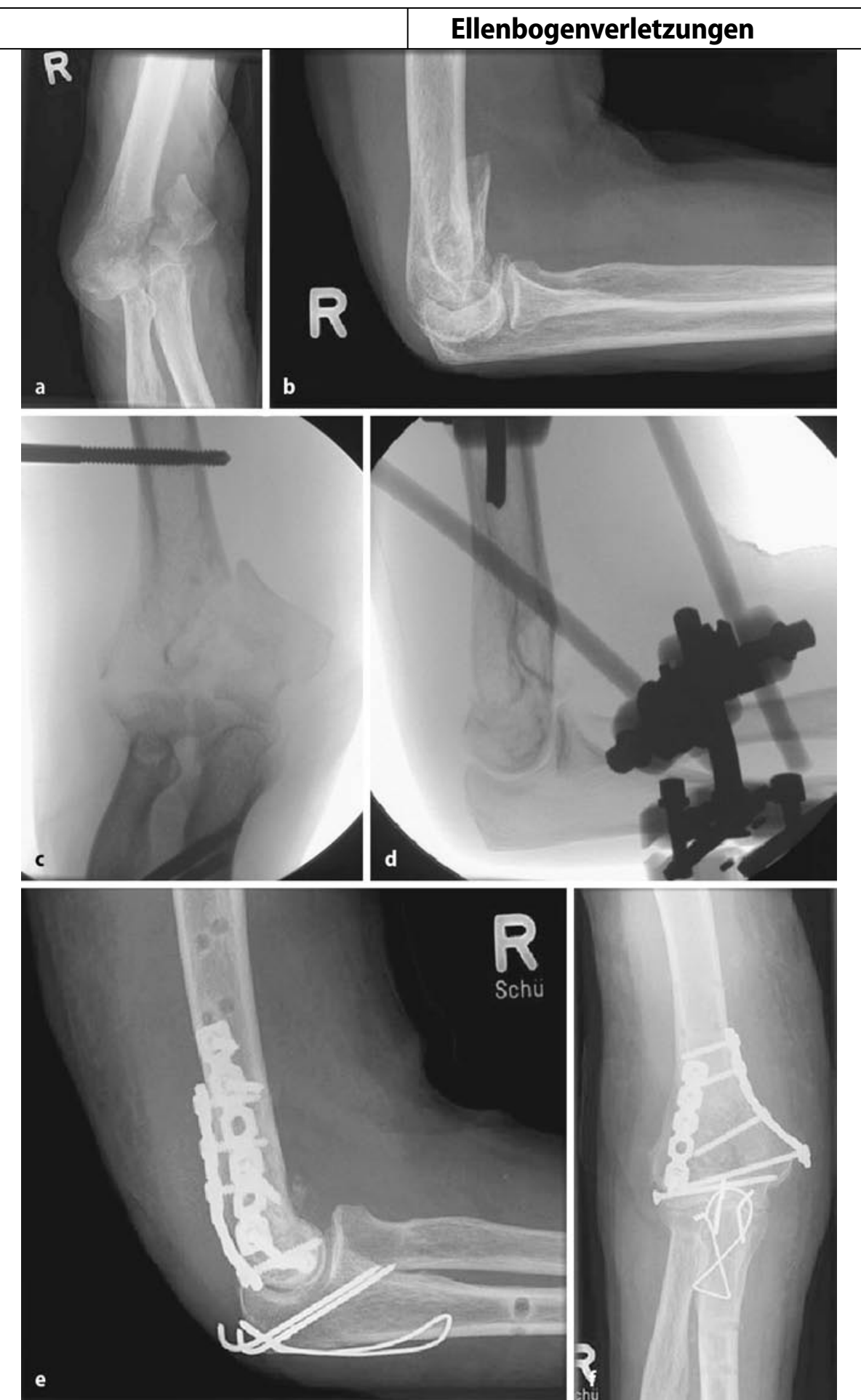

Abb. $2 \Delta$ 35-jähriger Patient mit C2-Fraktur im Rahmen eines Polytraumas nach Motorradunfall, a,b Unfallröntgenbilder in 2 Ebenen, $\mathbf{c}, \mathbf{d}$ primäre Fixation mit Fixateur externe, e,f nach 1 Woche offene Reposition und Plattenosteosynthese mit senkrecht zueinander stehenden Rekonstruktionsplatten am radialen und ulnaren Pfeiler

mer zu Immobilisationsschäden und Bewegungseinschränkungen führt.

Ziel der Behandlung sind:

- die anatomische Rekonstruktion der Gelenkfläche,

- eine Wiederherstellung der physiologischen Meta- und Diaphysenachsen und
Bei den Frakturen des Kindesalters wird kontrovers diskutiert, ob suprakondyläre Frakturen vom Typ 2 allein geschlossen reponiert werden können oder ob eine geschlossene Reposition mit anschließender perkutaner KirschnerDraht-Osteosynthese bessere Resultate ergibt. Die meisten vergleichenden Studien beziehen sich auf die chirurgische Fixation und die Konfiguration der Kirschner-Drähte, die gekreuzt medial nach lateral und umgekehrt oder allein von lateral eingebracht werden können $[9,17,19]$. Auch die antegrade Stabilisierung mit der ESIN-Methode (ESIN: elastisch-stabile intramedulläre Marknagelung) wird zunehmend publiziert.

Für die Erwachsenen haben sich die Gelenkrekonstruktionen mittels Plattenosteosynthese [Rekonstruktionsplatten, LCDCP $^{\star}$ (, limited contact dynamic compression plate“) oder $\mathrm{LCP}^{\star}$ (,locking compression plate"), anatomisch vorgeformte Platten] im rechten Winkel zueinander, d. h. dorsal auf den radialen Pfeiler und medial auf den ulnaren Pfeiler, durchgesetzt. Bei sehr schlechter Knochenqualität und bei weit epiphysär gelegenen Frakturen mit ausgedehnten Trümmerzonen hat sich in den letzten Jahren auch die primäre endoprothetische Versorgung bewährt [12].

Verglichen mit den konservativen immobilisierenden Behandlungsmethoden zeigte die operative Therapie in Level-3Studien $[7,34]$ folgende Vorteile:

- mehr exzellente und gute Ergebnisse

- hoher Prozentsatz an achsen- und gelenkgerechter Heilung

- 6-fach niedrigere Pseudarthroserate

- 4-fach niedrigeres Risiko für eine verzögerte Heilung

Für Kinder sind ebenfalls Level-2-Studien publiziert, mit folgenden Ergebnissen:

- Die operativen Behandlungen (primär geschlossene Reposition und perkutane Kirschner-Draht-Osteosynthese) scheinen der konservativen Therapie mit geschlossener Reposition und Ruhigstellung in Bezug auf klinisches Ergebnis und spätere Deformität überlegen zu sein. Allerdings resultieren bei der operativen Versorgung mehr lokale Infektionen $[1,6$, $16,30]$. 

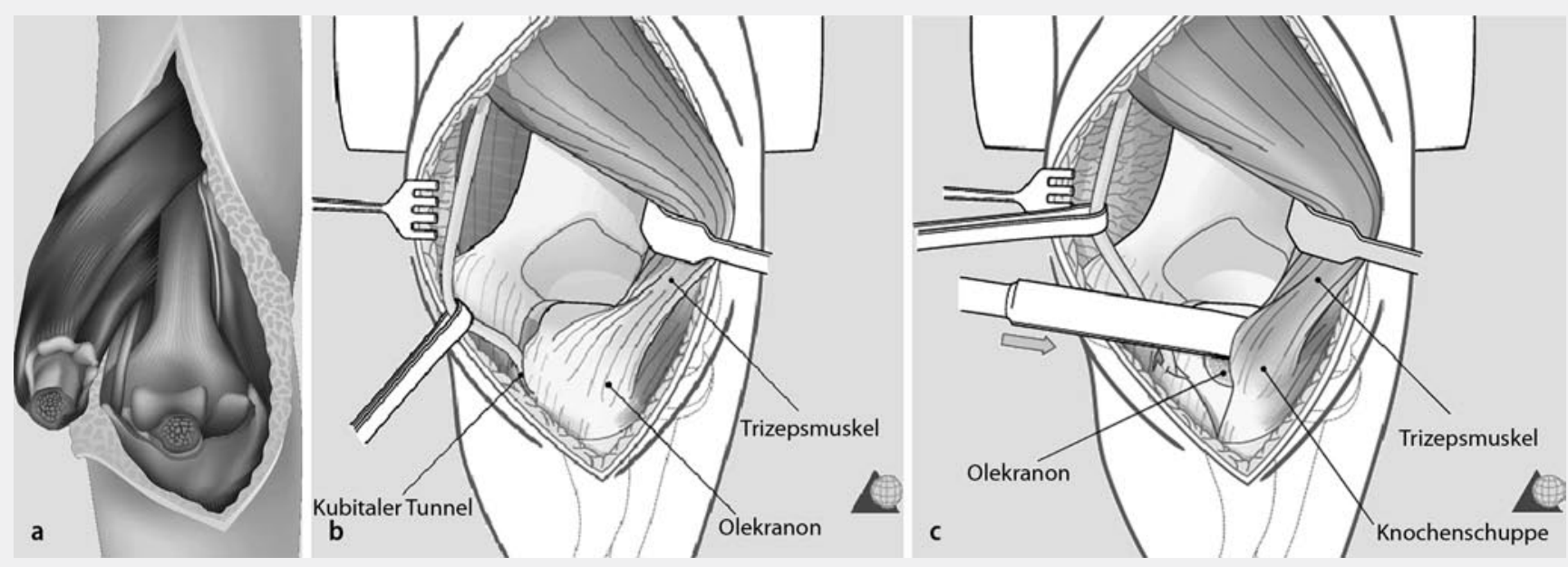

Abb. 3 \ Operative Zugangswege zum Ellenbogengelenk: a Olekranonosteotomie, b posteriorer Trizeps-on-Zugang („posterior triceps on approach“) nach Alonso-Llames [2], c posteriorer Trizepszugang („triceps-elevating approach“) nach Bryan u. Morrey [4]

Abb. 4 $\gg$ Operationstaktik: bei intraartikulären Frakturen zunächst Aufbau der Gelenkfläche mit Zugschrauben (a) oder in Kombination mit einer Stellschraube bei Zwischenfragmenten mit Defekt (b), dann Achsenreposition (c) und Plattenfixation mit dorsaler Platte radial (d) und medialer Platte ulnar (e)
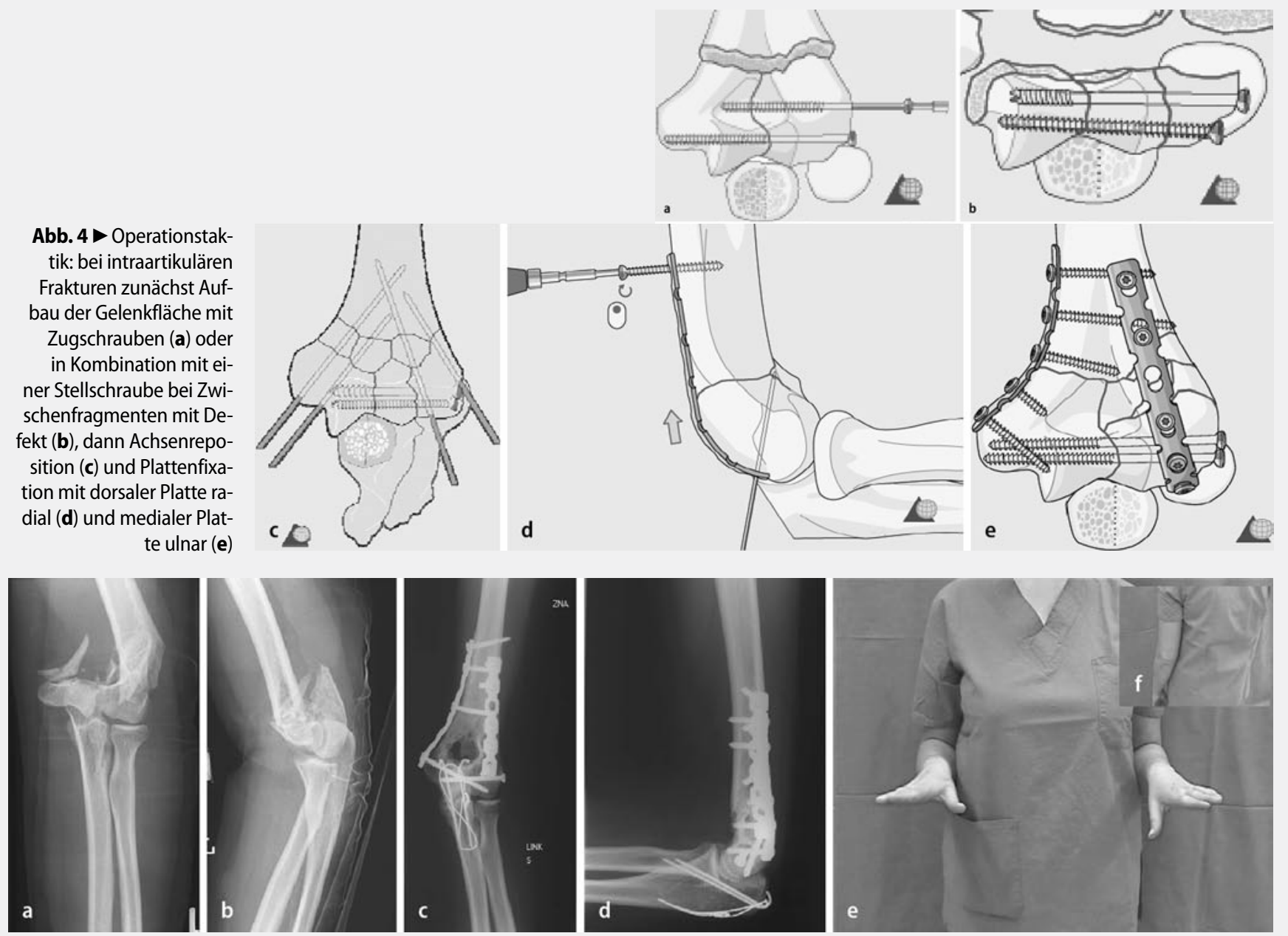

Abb. $5 \Delta$ 28-jährige Operationsschwester, a,b sehr weit distal gelegene C3 Fraktur nach Sturz auf Glatteis, c,d Ausheilungsbilder nach 1 Jahr, e,f Funktionsbilder

- Nach Osteosynthese werden mehr exzellente, gute und auch kosmetisch gute Ergebnisse im Flynn-Score $(82,5 \%$ gegenüber $62 \%)$ gesehen.
- Bei operativ behandelten dislozierten Frakturen ist das Risiko für die Entwicklung eines Cubitus varus im Vergleich zu den konservativ behandelten Brüchen halbiert.
- Für die Frakturen des lateralen Kondylus wurden signifikant niedrigere Raten für Pseudarthrosen und Repositionsverluste bei der operativen Behandlung berichtet ( $0-7 \%$ gegenüber 


\section{Ellenbogenverletzungen}
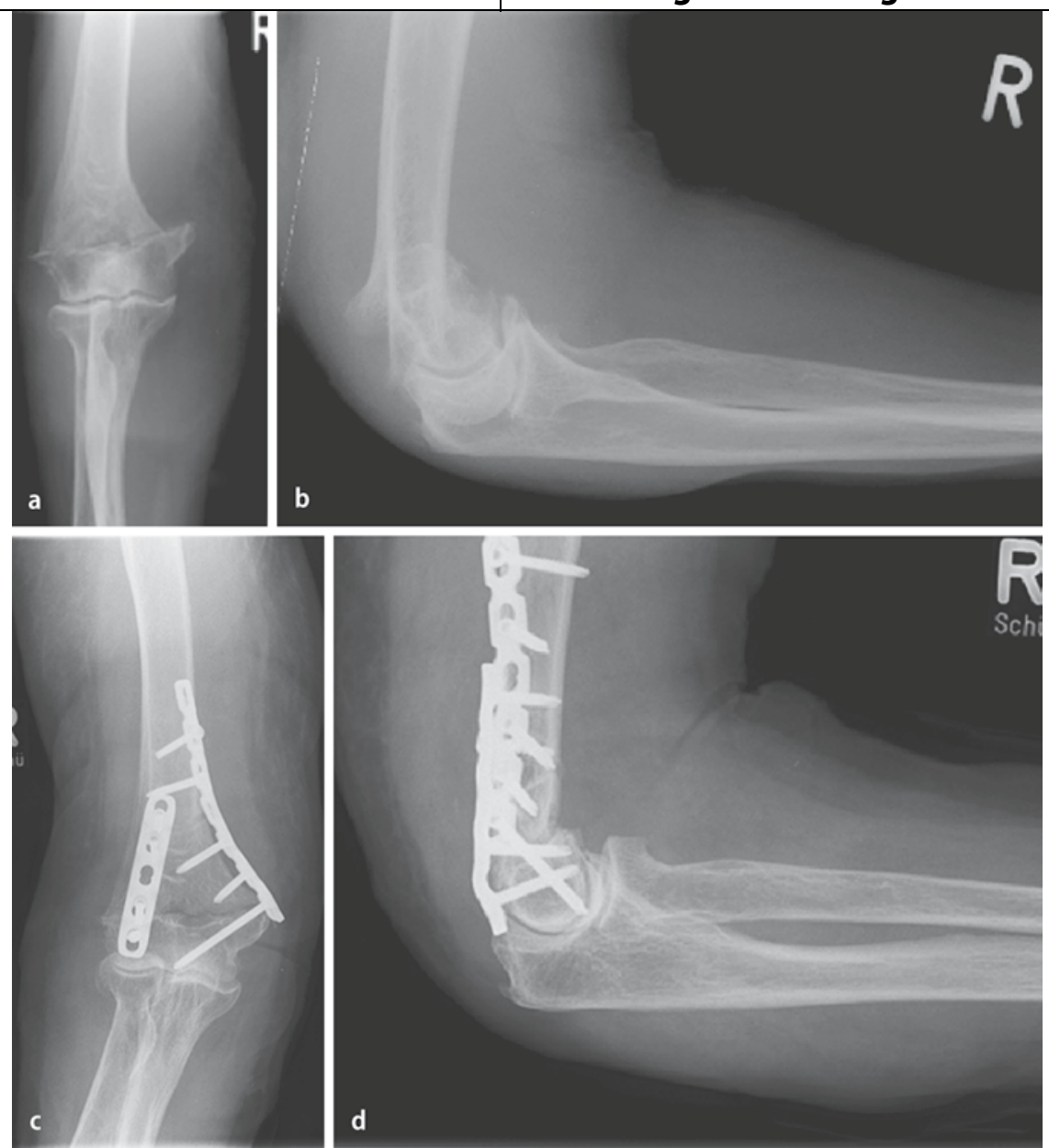

Abb. 6 \ 75-jährige Patientin mit wenig dislozierter Typ-A2-Verletzung $(\mathbf{a}, \mathbf{b})$ nach Sturz im Haushalt, primäre Ruhigstellung im Gips, $\mathbf{c}, \mathbf{d}$ Operation mit offener Reposition über dorsalen Trizepszugang nach Bryan u. Morrey [4], Fixation mit 2 winkelstabilen Platten mit möglichst langen distalen Schrauben
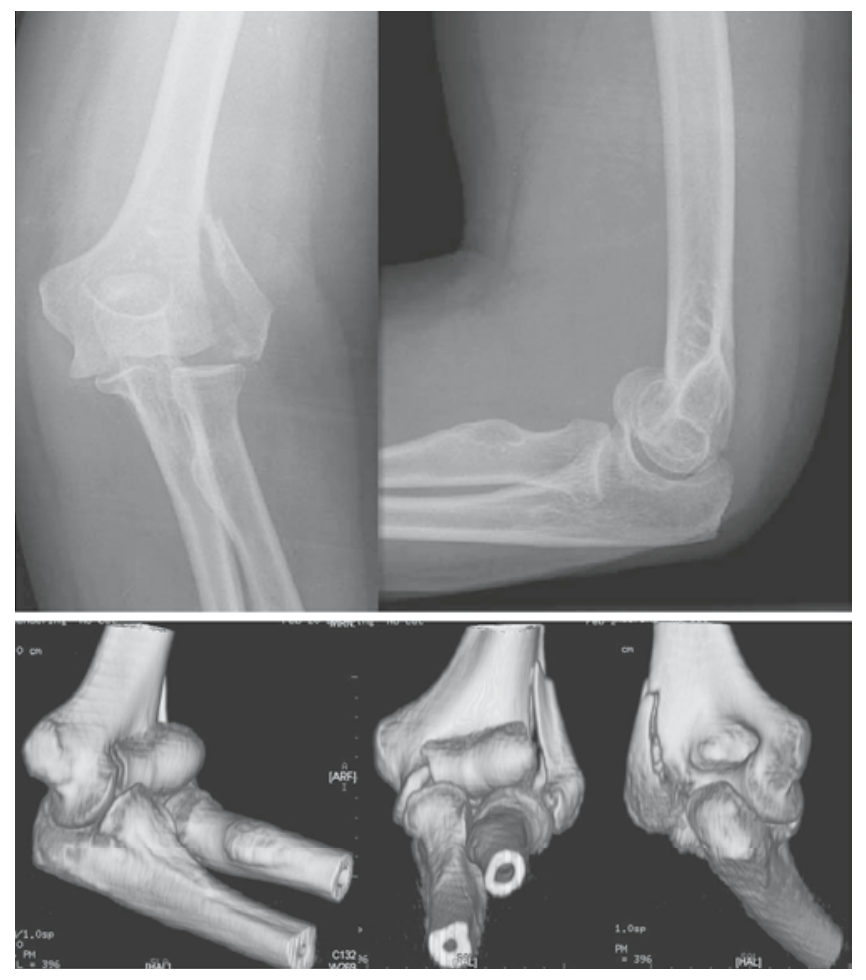

Abb. $7 \varangle$ Röntgen vs. Computertomographie: Typ-B3-Fraktur mit radialer Kondylenfraktur (Typ B2) bei 48jähriger Patientin nach Sturz vom Pferd, Ausmaß des gelenkseitigen Frakturverlaufs in dreidimensionaler CTDarstellung ersichtlich
30-40\%). Dies gilt auch für die nicht oder gering dislozierten Frakturen des lateralen Kondylus [3, 8, 10, 11, 15, $22,23,26,28,29,31]$.

\section{Zeitpunkt der Operation}

Die beste Versorgung ist immer die sofortige definitive Stabilisierung mit möglichst frühem Beginn der Bewegungstherapie $[5,12,13,14,20,27,28,32,33]$. Aus verschiedenen Gründen ist dies nicht immer und zu jeder Tages- und Nachtzeit zu realisieren. Unverzichtbar ist die sofortige Stabilisierung jedoch bei:

- offenen Frakturen

- Gefäß-/Nervenverletzungen

- Kompartmentsyndromen

- drohendem Weichteilschaden

- grober Dislokation

Im Notfall ist ein Gelenk überbrückender Fixateur externe vom Humerus auf die Ulna die Methode der Wahl. Der Eingriff kann in Rückenlage durchgeführt werden, ist ohne langen Zeitverlust planbar und durchführbar und schafft die Voraussetzungen für eine interne Osteosynthese (• Abb. 2).

Bei unbedrohten Weichteilen, geringer Dislokation und fehlenden Begleitverletzungen am Arm kann auch eine vorbereitende Immobilisation in einer umgreifenden Gipsschiene für eine Operationsvorbereitung ausreichend sein.

In der Praxis richtet sich der Zeitpunkt für die definitive Versorgung u. a. auch nach der Komplexizität der Verletzung, v. a. der Gelenkbeteiligung, der Kompetenz des Operateurs und der OP-Kapazität. Eine Endoprothesenimplantation benötigt eine gewisse Vorplanung und wird in der Regel nicht im Bereitschaftsdienst umgesetzt.

\section{Zugänge für die offene Versorgung}

Für die seltenen isolierten knöchernen Bandausrisse oder monokondylären Kondylenfrakturen können der laterale oder der mediale Zugang gewählt werden. Für die suprakondylären und Gelenkfrakturen bietet sich immer noch in erster Linie die Olekranonosteotomie mit V-förmiger Knocheninzision (Chevron-Osteotomie) und Hochschlagen des Trizeps- 
muskels nach proximal an. In der Regel erfolgen diese Eingriffe in Bauchlage über einer Rolle am Humerus. Möchte man eine Olekranonosteotomie vermeiden, sind der posteriore Trizeps-on-Zugang nach Alonso-Llames [2] mit Darstellung des N. ulnaris und radialem Beiseitehalten des Trizepsmuskels und der posteriore Trizepszugang nach Bryan u. Morrey [4] mit knöcherner Ablösung des Trizepsmuskels im Verbund mit einer Knochenschuppe am Olekranon und Belassen der distalen Fasern an der proximalen Ulna möglich. Letzterer wird auch für die Endoprothese bevorzugt.

Die beste Übersicht besteht u. E. bei der Olekranonosteotomie, die am Schluss des Eingriffs nach der Gelenkrekonstruktion und Stabilisierung der Fraktur mittels einer Zuggurtungsosteosynthese refixiert wird (• Abb.3).

\section{Operationstaktiken}

Die Operationsplanung erfolgt in der Regel anhand der Röntgen- und CT-Aufnahmen (0 Abb. 7).

Nach übersichtlicher Darstellung des N. ulnaris wird dieser angeschlungen und über ein langstreckiges Release mobilisiert. Nach Freilegung der Frakturanteile wird das Gelenk rekonstruiert. Bei den mehrfragmentären vollständigen Gelenkfrakturen stellt dies die schwierigste Aufgabe dar. Wird der Arm in Bauchlage über einer Rolle gelagert, lässt sich die Fraktur in der Meta- und Diaphyse durch Extension und Flexion sowie Valgisierung und Varisierung sehr gut einstellen. In der Regel ist bei $90^{\circ}$ Flexion und hängendem Arm die spontane Ausrichtung möglich. Die Gelenkfragmente lassen sich allerdings hierdurch nicht beliebig manipulieren. Je nach Gegebenheit wird die Gelenkrolle entweder mit einer von lateral nach medial verlaufenden Zugschraube oder mehreren Kleinfragmentschrauben (im Falle des intermediären Fragments auch mittels resorbierbarer Stifte) rekonstruiert und anschließend zunächst mit kräftigen Kirschner-Drähten $(2,0 \mathrm{~mm})$ an die Metaphyse angeheftet. Gelingt der Aufbau mit diesem Verfahren nicht, kann man bei der Rekonstruktion des Gelenks von einem Pfeiler, der noch eine günstige Referenzzone zeigt, beginnen. Wenn die
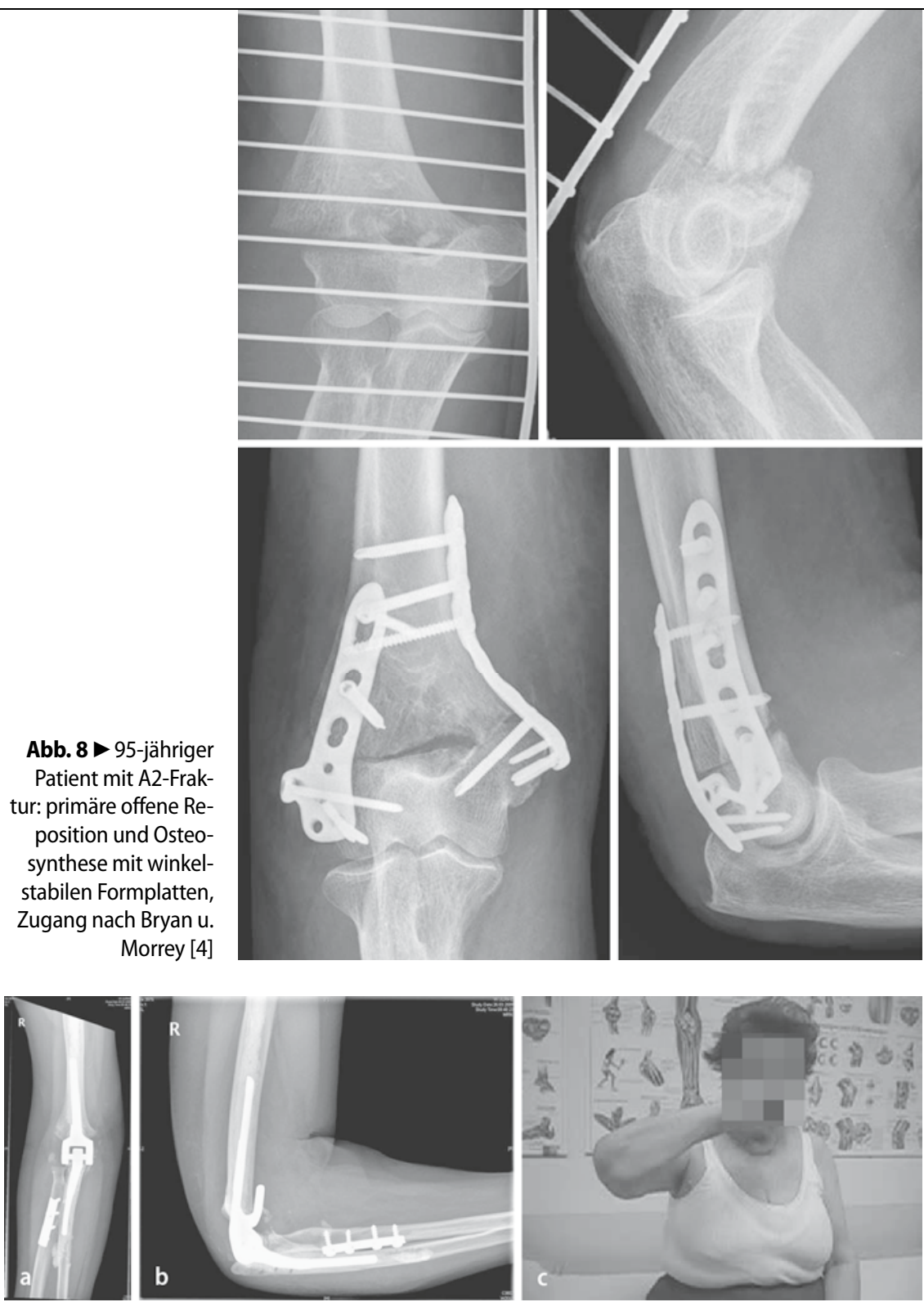

Abb. $9 \triangle$ 66-jährige Patientin, 6 Monate nach distaler Humerusfraktur und Osteosyntheseversagen: $\mathbf{a}, \mathbf{b}$ nach Kondylenentfernung eingebrachte Prothese mit Abstützung an der Metaphyse (ConradMorrey), c gute Funktion nach 3 Monaten

Achsen gesichert sind, werden die Platten wahlweise radial oder ulnar senkrecht zueinander angebracht (• Abb. 4). Speziell bei den epiphysär gelegenen Frakturen ist es hierbei dringend geboten, möglichst lange Schrauben durch die distalen Plattenlöcher einzubringen ( $\bullet$ Abb.5). Bei schlechter Knochenqualität sollten zudem möglichst auch winkelstabile Implantate verwendet werden (- Abb. 6). Hier scheinen polyaxial winkelstabile Implantate von Vorteil zu sein, da sie durch die größere Variabilität der möglichen Schraubenlagen dem Operateur mehr Freiheiten bieten. In der Regel gelingt es, eine sofortige Bewegungsstabilität herzustellen.

\section{Implantate}

Experimentelle Untersuchungen von Körner et al. [18] ergaben, dass winkelstabile Implantate am distalen Humerus speziell bei manifester Osteoporose hilfreich sein können. Sie gehen mit einer Zunahme der Steifigkeit bei $90^{\circ}$ Flexion im Ellenbogen einher. Bei guter Knochenqualität lässt sich diesbezüglich jedoch kein Unterschied zu den üblichen Implantaten bei der klassischen Implantatlage radial/ 

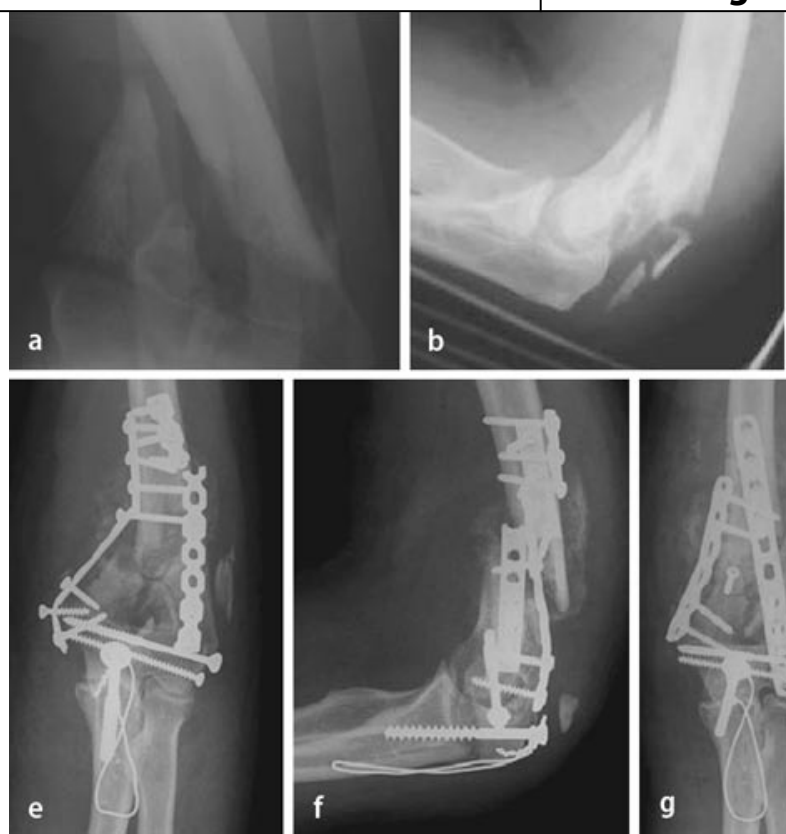

b

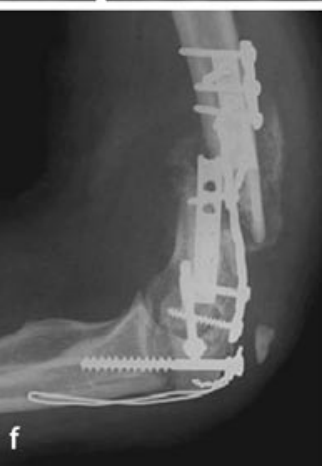

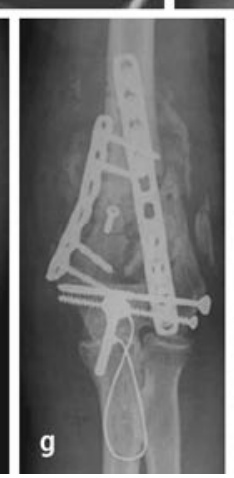
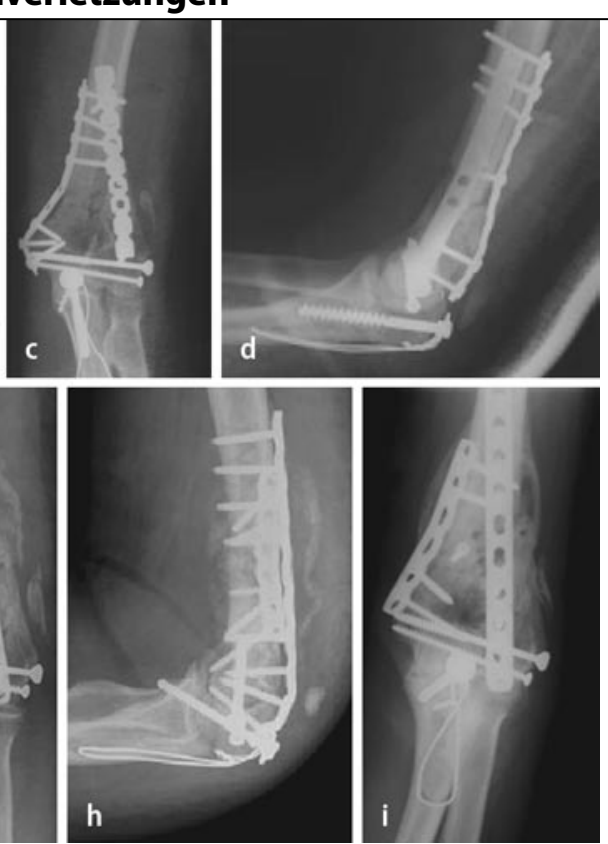

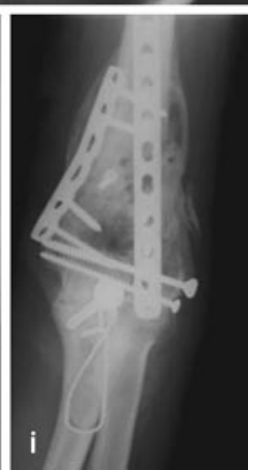

Abb. $10<$ Verlauf mit Komplikation: 71 Jahre alter Patient mit 13C2-Fraktur $(\mathbf{a}, \mathbf{b})$ nach Sturz auf den Ellenbogen aus dem Stand, sofortige offene Reposition und Osteosynthese mit Drittelrohrplatte ulnar und Rekoplatte radial, mit Zugschraube und Zuggurtung fixierte Olekranonosteotomie $(\mathbf{c}, \mathbf{d})$, nach 6 Wochen Plattenbrüche $(\mathbf{e}, \mathbf{f})$, nach 2 Monaten Reosteosynthese mit winkelstabilen Platten (LCP, $\mathbf{g})$, Ausheilung nach 6 Monaten $(\mathbf{h}, \mathbf{i})$ dorsal und ulnar/medial herausarbeiten. Drittelrohrplatten sind allenfalls für Epikondylenfrakturen oder monokondyläre Frakturen geeignet ( $\mathbf{A}$ Abb. 7). Isolierte Kirschner-Draht-Osteosynthesen beim Erwachsenen sind auch bei extraartikulären Frakturen nicht für eine Stabilisierung geeignet. Auch diese Verletzungen erfordern eine übungsstabile Versorgung (• Abb. 8).

Das Plattendesign soll der Knochenoberfläche möglichst angepasst sein und benötigt eine gewisse Stärke. Diese Kriterien erfüllen die Drittelrohrplatten in der Regel nicht. Eine Multiaxialität ist bei den Gelenkfrakturen ebenso von Vorteil, wie die Möglichkeit der Verankerung möglichst vieler Schrauben in den distalen Fragmenten.

Winkelstabile Implantate haben bei sehr weit distal gelegenen Frakturen, suprakondylären Trümmerzonen sowie beim osteoporotischen Knochen u. E. den Vorteil einer höheren Stabilität. Sie sind auch für Reosteosynthesen gut geeignet und lassen sich problemlos mit Kompressionsplatten kombinieren.

Für die primäre Endoprothetik kommt in der Regel der Erhalt der Kondylen nicht in Frage. Deswegen sind für die Behandlung in erster Linie Endoprothesen, die sich distal meta- oder diaphysär abstützen, ein adäquates Mittel.

Die sekundäre Endoprothetik bei Versagen oder schlechtem Ergebnis der Osteosynthese kann auf einen Kondylenerhalt zielen. Jedoch sind auch bei diesem Verfahren hin und wieder die augenscheinlich verheilten Frakturen nicht so fest konsolidiert, dass sie eine ausreichende Fixierung der Kondylen erhaltenden Implantate gewährleisten. In diesen Fällen muss sich die endoprothetische Versorgung dem intraoperativen Befund unterordnen und es muss ggf. auf einen anderen Implantattyp umgestiegen werden (• Abb. 9).

\section{Komplikationen}

Insgesamt gesehen sind Komplikationen nach Hochrasanztraumen häufiger. Neben primären Nervenverletzungen resultieren nicht selten auch sekundäre, iatrogene Nervenläsionen durch die Freilegung und das Beiseitehalten der Nerven im Rahmen der Manipulationen zur Frakturreposition. Intraoperative Gefäßläsionen sind selten. Sie lassen sich meist auf eine Begleitverletzung im Rahmen eines Hochrasanztraumas zurückführen. Gelegentlich treten sie auch bei Luxationsverletzungen auf.

Pseudarthrosen sind v. a. bei insuffizienter Fixation oder Implantatverwendung zu erwarten ( $\mathbf{A b b}$. 10). Auch belassene Achsfehlstellungen prädestinieren hierfür.

Heterotope Ossifikationen gehen häufig auf primär belassene kleine knöcherne Absprengungen und Fragmente in der Trümmerzone des Bruchs zurück und können zu Bewegungseinschränkungen v. a. in der Flexion führen. Sie können allerdings auch Ausdruck einer verbliebenen Instabilität im Frakturniveau sein. Bei Disposition zu heterotopen Ossifikationen (Anamnese nach Hüftendoprothesenimplantation) empfiehlt sich eine 14tägige Prophylaxe mit 50 mg Indometacin 1-mal täglich.

\section{Bewegungseinschränkung}

In den seltensten Fällen können die vollen Bewegungsausmaße am Ellenbogen wieder erreicht werden. Ein sehr gutes Ergebnis bringt eine Beweglichkeit von o/10/130 bei freier Rotation. Diese ist im Übrigen in den seltensten Fällen eingeschränkt. Vor allem die Beugung des Ellenbogens ist für die Durchführung von Alltagsbewegungen wie Essen, Trinken, Haare kämmen und die Zahnpflege wichtig. Anlässlich von individuell zu planenden Metallentfernungen können Arthrolysen vorgenommen werden, die einer besonderen Operationstechnik und Nachbehandlung bedürfen.

\section{Fazit}

Die distalen Humerusfrakturen stellen ein zunehmendes Problem bei der älteren Bevölkerung mit schlechter Knochenqualität dar. Wichtig für das Verständnis der Frakturbehandlung ist die grundsätzliche Unterscheidung in Hochrasanz- und Niedrigenergiemechanis- 
men. Während die Hochrasanztraumen in der Regel mehr Begleitkomplikationen verursachen, erhöhen Niedrigrasanztraumen häufig die Schwierigkeit der übungsstabilen Refixation. Eine zentrale Bedeutung kommt der primären Weichteilentlastung bei dislozierten Frakturen zu. Sie kann im Wesentlichen mit dem Fixateur externe erreicht werden, wenn eine primäre Operation aus medizinischen oder logistischen Gründen nicht durchführbar ist. Das oberste Ziel ist die exakte stufenfreie Rekonstruktion der Gelenkfläche bei ligamentärer Stabilität in Verbindung mit der Wiederherstellung der physiologischen dia- und metaphysären Achsenverhältnisse. Die durch die Operation erreichte Stabilität sollte immer eine sofortige Bewegungstherapie des Ellenbogens ermöglichen. Falls dies durch eine Osteosynthese nicht gewährleistet werden kann, können endoprothetische Versorgungen v. a. beim älteren Patienten zu guten Ergebnissen führen. Die Sekundärendoprothetik nach Osteosynthesen ist $u$. E. ebenfalls eine erfolgreiche Methode, um eine zeitnahe Rehabilitation und Wiederherstellung bei älteren Patienten zu erreichen.

\section{Korrespondenzadresse \\ Prof. Dr. F. Bonnaire}

Klinik für Unfall-, Wiederherstellungs-

und Handchirurgie,

Städtisches Klinikum Dresden-Friedrichstadt, Akademisches Lehrkrankenhaus, TU Dresden, Friedrichstraße 41, 01067 Dresden bonnaire-fe@khdf.de

Interessenkonflikt. Keine Angaben

\section{Literatur}

1. Ababneh M, Shannak A, Agabi S, Hadidi S (1998) The treatment of displaced supracondylar fractures of the humerus in children. A comparison of three methods. Int Orthop 22:263-265

2. Alonso-Llames M (1972) Bilaterotricipital approach to the elbow. Its application in the osteosynthesis of supracondylar fractures of the humerus in children. Acta Orthop Scand 43(6):479-490

3. Aronson DC, Vollenhoven E van, Meeuwis JD (1993) K-wire fixation of supracondylar humeral fractures in children: results of open reduction via a ventral approach in comparison with closed treatment. Injury 24:179-181

4. Bryan RS, Morrey BF (1982) Extensive posterior exposure of the elbow. A triceps-sparing approach. Clin Orthop 166:188-192
5. Carmichael KD, Joyner K (2006) Quality of reduction versus timing of surgical intervention for pediatric supracondylar humerus fractures. Orthopedics 29:628-632

6. Celiker O, Pestilci FI, Tuzuner M (1990) Supracondylar fractures of the humerus in children: analysis of the results in 142 patients. J Orthop Trauma 4:265-269

7. Chen RS, Liu CB, Lin XS et al (2001) Supracondylar extension fracture of the humerus in children. $\mathrm{Ma}$ nipulative reduction, immobilisation and fixation using a U-shaped plaster slab with the elbow in full extension. J Bone Joint Surg Br 83:883-887

8. Cramer KE, Devito DP, Green NE (1992) Comparison of closed reduction and percutaneous pinning versus open reduction and percutaneous pinning in displaced supracondylar fractures of the humerus in children. J Orthop Trauma 6:407-412

9. Davis RT, Gorczyca JT, Pugh K (2000) Supracondylar humerus fractures in children. Comparison of operative treatment methods. Clin Orthop 376:49-55

10. Buys Roessingh AS de, Reinberg O (2003) Open or closed pinning for distal humerus fractures in children? Swiss Surg 9:76-81

11. Foead A, Penafort R, Saw A, Sengupta S (2004) Comparison of two methods of percutaneous pin fixation in displaced supracondylar fractures of the humerus in children. J Orthop Surg (Hong Kong) 12:76-82

12. Frankle MA, Herscovici D Jr, DiPasquale TG et al (2003) A comparison of open reduction and internal fixation and primary total elbow arthroplasty in the treatment of intraarticular distal humerus fractures in women older than age 65. J Orthop Trauma 17:473-480

13. Gupta N, Kay RM, Leitch K et al (2004) Effect of surgical delay on perioperative complications and need for open reduction in supracondylar humerus fractures in children. J Pediatr Orthop 24:245248

14. lyengar SR, Hoffinger SA, Townsend DR (1999) Early versus delayed reduction and pinning of type III displaced supracondylar fractures of the humerus in children: a comparative study. J Orthop Trauma 13:51-55

15. Kaewpornsawan K (2001) Comparison between closed reduction with percutaneous pinning and open reduction with pinning in children with closed totally displaced supracondylar humeral fractures: a randomized controlled trial. J Pediatr Orthop B 10:131-137

16. Kennedy JG, El Abed K, Soffe K et al (2000) Evaluation of the role of pin fixation versus collar and cuff immobilisation in supracondylar fractures of the humerus in children. Injury 31:163-167

17. Khan MS, Sultan S, Ali MA et al (2005) Comparison of percutaneous pinning with casting in supracondylar humeral fractures in children. J Ayub Med Coll Abbottabad 17:33-36

18. Körner J, Diederichs G, Arzdorf M et al (2004) A biomechanical evaluation of methods of distal humerus fracture fixation using locking compression plates versus conventional reconstruction plates. J Orthop Trauma 18:286-293

19. Launay F, Leet Al, Jacopin S et al (2004) Lateral humeral condyle fractures in children: a comparison of two approaches to treatment. J Pediatr Orthop 24:385-391

20. Mehlman CT, Strub WM, Roy DR et al (2001) The effect of surgical timing on the perioperative complications of treatment of supracondylar humeral fractures in children. J Bone Joint Surg Am 83A:323-327
21. Melton LJ 3rd, Amadio PC, Crowson CS, O'Fallon WM (1998) Long-term trends in the incidence of distal forearm fractures. Osteoporos Int 8:341-348

22. Oh CW, Park BC, Kim PT et al (2003) Completely displaced supracondylar humerus fractures in children: results of open reduction versus closed reduction. J Orthop Sci 8:137-141

23. Ozkoc G, Gonc U, Kayaalp A et al (2004) Displaced supracondylar humeral fractures in children: open reduction vs. closed reduction and pinning. Arch Orthop Trauma Surg 124:547-551

24. Robinson CM, Hill RM, Jacobs N et al (2003) Adult distal humeral metaphyseal fractures: epidemiology and results of treatment. J Orthop Trauma 17:38-47

25. Ruedi T, Murphy WM (2000) AO principles of fracture management, vol 1. AO Publishing, New York

26. Shamsuddin SA, Penafort R, Sharaf I (2001) Crossed-pin versus lateral-pin fixation in pediatric supracondylar fractures. Med J Malaysia [Suppl D] 56:38-44

27. Sibinski M, Sharma H, Bennet GC (2006) Early versus delayed treatment of extension type-3 supracondylar fractures of the humerus in children. J Bone Joint Surg Br 88:380-381

28. Sibinski M, Sharma H, Sherlock DA (2006) Lateral versus crossed wire fixation for displaced extension supracondylar humeral fractures in children. Injury 37:961-965

29. Solak S, Aydin E (2003) Comparison of two percutaneous pinning methods for the treatment of the pediatric type III supracondylar humerus fractures. J Pediatr Orthop B 12:346-349

30. Sutton WR, Greene WB, Georgopoulos G, Dameron TB Jr (1992) Displaced supracondylar humeral fractures in children. A comparison of results and costs in patients treated by skeletal traction versus percutaneous pinning. Clin Orthop 278:81-87

31. Topping RE, Blanco JS, Davis TJ (1995) Clinical evaluation of crossed-pin versus lateral-pin fixation in displaced supracondylar humerus fractures. J Pediatr Orthop 15:435-439

32. Waddell JP, Hatch J, Richards R (1988) Supracondylar fractures of the humerus - results of surgical treatment. JTrauma 28:1615-1621

33. Walmsley PJ, Kelly MB, Robb JE et al (2006) Delay increases the need for open reduction of typeIII supracondylar fractures of the humerus. J Bone Joint Surg Br 88:528-530

34. Zagorski JB, Jennings JJ, Burkhalter WE, Uribe JW (1986) Comminuted intraarticular fractures of the distal humeral condyles. Surgical vs. nonsurgical treatment. Clin Orthop 202:197-204 